\title{
The icy side of Frosty Leo
}

\author{
B. Lopez ${ }^{1}$, P. G. Tuthill ${ }^{2}$, W. C. Danchi ${ }^{3,4}$,J. D. Monnier ${ }^{5}$, and G. Niccolini ${ }^{1}$ \\ 1 Observatoire de la Côte d'Azur, Département Fresnel UMR 6528, BP 4229, 06034 Nice Cedex 4, France \\ 2 School of Physics, University of Sydney, NSW 2006, Australia \\ 3 NASA Goddard Space Flight Center, Infrared Astrophysics, Code 685, Greenbelt, HD 20771, USA \\ 4 Space Sciences Laboratory, University of California, Berkeley, Berkeley, CA 94720-7450, USA \\ 5 Smithsonian Astrophysical Observatory, MS42, 60 Garden Street, Cambridge, MA 02138, USA
}

Received 31 July 2000 / Accepted 12 June 2001

\begin{abstract}
An imaging study of the bipolar nebula Frosty Leo is presented. In particular, we have studied the effects of spatial distribution of $\mathrm{H}_{2} \mathrm{O}$ ice on the circumstellar environment. Using the Keck telescope with the NIRC infrared camera, we have recovered images at a wavelength of $3.08 \mu \mathrm{m}$ within the prominent (attenuation of about $5 \mathrm{mag}$ ) water-ice absorption band, and in the adjacent quasi-continuum at $1.6 \mu \mathrm{m}, 2.2 \mu \mathrm{m}$, and $3.3 \mu \mathrm{m}$. While the well-known bipolar structure appears quite symmetrical in all quasi-continuum images, the Northern lobe seems to be almost totally extinguished (by a factor of $\gtrsim 10$ ) compared to the Southern one in images at $3.08 \mu \mathrm{m}$. This suggests a much greater concentration of ice between the observer and the Northern lobe. The question of the physical structure of the nebula has been addressed with the use of numerical radiative transfer simulations which have been able to reproduce the general behavior of the images and of earlier spectral data. From this, we deduce and/or confirm several parameters of interest: the typical dust grain size, the dust density distribution in the envelope, and the abundance of water ice. Models were produced with physically symmetrical lobes in which some one-sided extinction was produced by an effect related to the observer's viewing angle. However, in order to reproduce the extreme extinction observed, more complicated scenarios, involving asymmetry with respect to the plane of the disk, were needed. Additional imaging observations were conducted at $2.26 \mu \mathrm{m}$ (where the system is bright) with the intent to recover high-angular-resolution information. Data sets consisted of one hundred of rapid-exposure data frames from which images were recovered using a shift-and-add algorithm. Although these images did contain structures at the diffraction limit of the telescope ( $\sim 50$ milliarcsec), no evidence for binarity as reported by Roddier et al. (1995) from adaptive optical studies was found within the dynamical range obtained.
\end{abstract}

Key words. radiative transfer - methods: numerical - methods: observational - techniques: interferometric stars: mass loss - stars: AGB and post-AGB

\section{Introduction}

The evolution of intermediate mass (from 1 to $\sim 8 M_{\odot}$ ) stars from the time they leave the asymptotic giant branch (AGB) until they form planetary nebulae (PNe) is gradually being understood. Post-AGB objects are surrounded by envelopes of gas and dust which often display a bipolar morphology, strikingly illustrated in such textbook examples as the Red Rectangle, the Egg nebula and Frosty Leo.

Frosty Leo, christened after the detection of $\mathrm{H}_{2} \mathrm{O}$ ice in its circumstellar shell (Forveille et al. 1987; Likkel et al. 1987; Rouan et al. 1988), is one of the best examples of the brief transitional phase between the AGB and PNe. This object is particularly interesting due to the bipolar jets thought to arise in the binary system

Send offprint requests to: B. Lopez,

e-mail: lopez@obs-nice.fr
(Roddier et al. 1995) at the heart of the nebula. With active mass loss still taking place at a rate of about $2 \times 10^{-6}[D / \mathrm{kpc}] M_{\odot} \mathrm{yr}^{-1}$ (Forveille et al. 1987), Frosty Leo is an excellent candidate for study of stellar evolution during the early post-AGB sequence.

Its distance is poorly constrained (a $1 \mathrm{kpc}$ lower limit was set by Mauron et al. 1989). The terminal velocity of the gas has been obtained from $\mathrm{CO}$ measurements to be $25 \mathrm{~km} \mathrm{~s}^{-1}$ (Forveille et al. 1987).

The lifetime of a star on the post-AGB sequence before it becomes a planetary nebula (photoionizing hydrogen in the envelope when $T_{\text {eff }}$ is greater than $20000 \mathrm{~K}$ ) is rather short. Blöker (1995) computes that a star with an initial main sequence mass of $3 M_{\odot}$ is expected to have a postAGB phase lasting only a few thousand years; hence the relative rarity of post-AGB objects. Frosty Leo, one of the nearest and brightest post-AGB stars, has a number of 
speculiarities that we detail below: a) firstly, its bipolar lobes are clearly separated in the plane of the sky with a possible binary core; b) secondly, it is thought to occupy a place at the middle of its post-AGB life; and c) thirdly, the presence of $\mathrm{H}_{2} \mathrm{O}$ ice is firmly established in the circumstellar shell.

a) Bipolarity in post-AGB nebulae is usually interpreted in terms of an equatorial concentration (disk or toroid) of dust which absorbs the stellar radiation in the equatorial region, while allowing radiation to escape along the poles. Radiative transfer calculations based on this geometry are able to successfully simulate bipolar nebulae (see for example Yusef-Zadeh et al. 1984; Lopez \& Perrin 2000), particularly for the case where the line of sight lies on or near the equatorial plane. However the underlying cause for bipolarity in post-AGB nebulae is still debated. Morris (1981) suggests that the bipolar structure results from the interaction of a mass-losing red giant and a companion star. The companion gravitationally deflects some of the red giant wind allowing formation of the disk (Mastrodemos \& Morris 1998, 1999). In addition, an accretion flow onto the companion star may result in the creation of a fast polar outflow or jet (Morris 1987).

Such connections between bipolar structures and binarity have been strengthened by recent observations. Spectroscopic observations of the central source of the Red Rectangle nebula (Van Winckel et al. 1995) supports the binary model, as does the finding of binarity within the central source in Frosty Leo (Roddier et al. 1995). Also possibly related to binary models is the presence of a high velocity polar outflow, first inferred from visible images of polar knots about $12^{\prime \prime}$ from the center of the nebula (Morris \& Reipurth 1990), and later as bipolar jets seen at high resolution (Roddier et al. 1995).

b) The mass loss activity of a late type star increases dramatically during the AGB phase of stellar evolution. However, mass loss rate variations for different stages of late stellar evolution are not well characterized or understood (Lafon \& Berruyer 1991). During the post-AGB phase, Alves \& Hoard (1996) suggest a classification based on the dynamical evolution of the envelope as follows. "Young post-AGB" systems exhibit central stars which are totally obscured by thick disks of dust (prototypical examples include the Egg Nebula and $\mathrm{OH} 231.8+4.2$ ). "Middle-aged post-AGB" systems (such as Frosty Leo) are those in which the circumstellar material has been significantly dispersed to reveal the central object. "Old postAGB" systems (e.g. M2-9 and Mz-3) are seen as reflection and emission nebulae with the central star(s) photoionizing their surrounding environment.

c) $\mathrm{H}_{2} \mathrm{O}$ ice has been detected in the circumstellar envelopes of a number of evolved stars. Characteristic absorption bands at $3.1 \mu \mathrm{m}$ and/or $12 \mu \mathrm{m}$ have been observed in OH231.8+4.2 by Soifer et al. (1981), in OH32.80.3 by Roche \& Aitken (1984), in M1-92 by Eiroa et al. (1983) and in Frosty Leo by Forveille et al. (1987) and by Rouan et al. (1988). Dust particles are thought to be large with a radius of about $1 \mu \mathrm{m}$ and with an ice mass fraction of $\sim 40 \%$ (Forveille et al. 1987; Rouan et al. 1988). Omont et al. (1990) show spectra with two emission features at 44 and $62 \mu \mathrm{m}$. When compared to laboratory data, the observed emission profiles appear to match that of crystalline ice, amorphous ice is precluded because its emissivity does not show the observed secondary maximum at $62 \mu \mathrm{m}$.

In the present work, we report near-infrared images obtained with the Keck telescope. A variety of chopping, nodding and shift-and-add techniques were used as described in more detail in the following section. The primary objective of the observations was to obtain high quality images at a wavelength centered on the deep $3.1 \mu \mathrm{m}$ ice feature, together with comparative images in the neighboring continuum at 1.6, 2.2 and $3.3 \mu \mathrm{m}$. In addition, the high resolution obtainable at the diffraction limit of the $10 \mathrm{~m}$ Keck aperture has permitted a study of the inner regions of the nebula at very fine scales. In Sect. 3 we propose a non-spherical radiative transfer model of the nebula which reproduces the general behaviour of the near-infrared images and the broad band spectrum. Further discussion and comparison with earlier work (Robinson et al. 1992) under the assumptions of spherical geometry is given in Sect. 4 .

\section{Observations}

Observations were carried out using the Near InfraRed Camera (NIRC) (Matthews \& Soifer 1994) on the Keck I telescope on the nights of 7 and 9 June 1998. The observing wavelength was set using internal interference filters, giving access to a number of set wavebands within the near-IR. The observing strategy for each observation was customized to yield the optimum performance from the camera and telescope, as described in more detail below.

The primary observations carried out for this study were made with the camera in a "standard" observing configuration, which yielded a $256 \times 256$ frame with a fieldof-view $38^{\prime \prime} 4$ on a side. At this plate scale, the diffraction pattern from the Keck aperture was not Nyquist sampled, so it was not possible to attain the highest spatial resolution from these data sets. The "standard" configuration did, however, allow the imaging of the extended Frosty Leo nebula with very high dynamic range, even in the ice band near $3.1 \mu \mathrm{m}$, where the flux drops by almost two orders of magnitude. Integrations typically consisted of 100 co-added frames, each with the short integration time of some $\sim 140 \mathrm{~ms}$ to prevent saturation of the array due to scattered ambient and sky radiation. In order to ensure reliable flat-fielding and resilience against bad pixels and other camera-related artifacts, the image of the nebula was moved to a number of different locations on the array. The final images, presented in Fig. 1, are composites of many individually calibrated raw frames.

Circumstellar nebulosity can be seen to be present in maps made at all near-IR filter bands with substantial differences of morphology being readily apparent for the four different filters. The $H$-band image, while clearly showing 


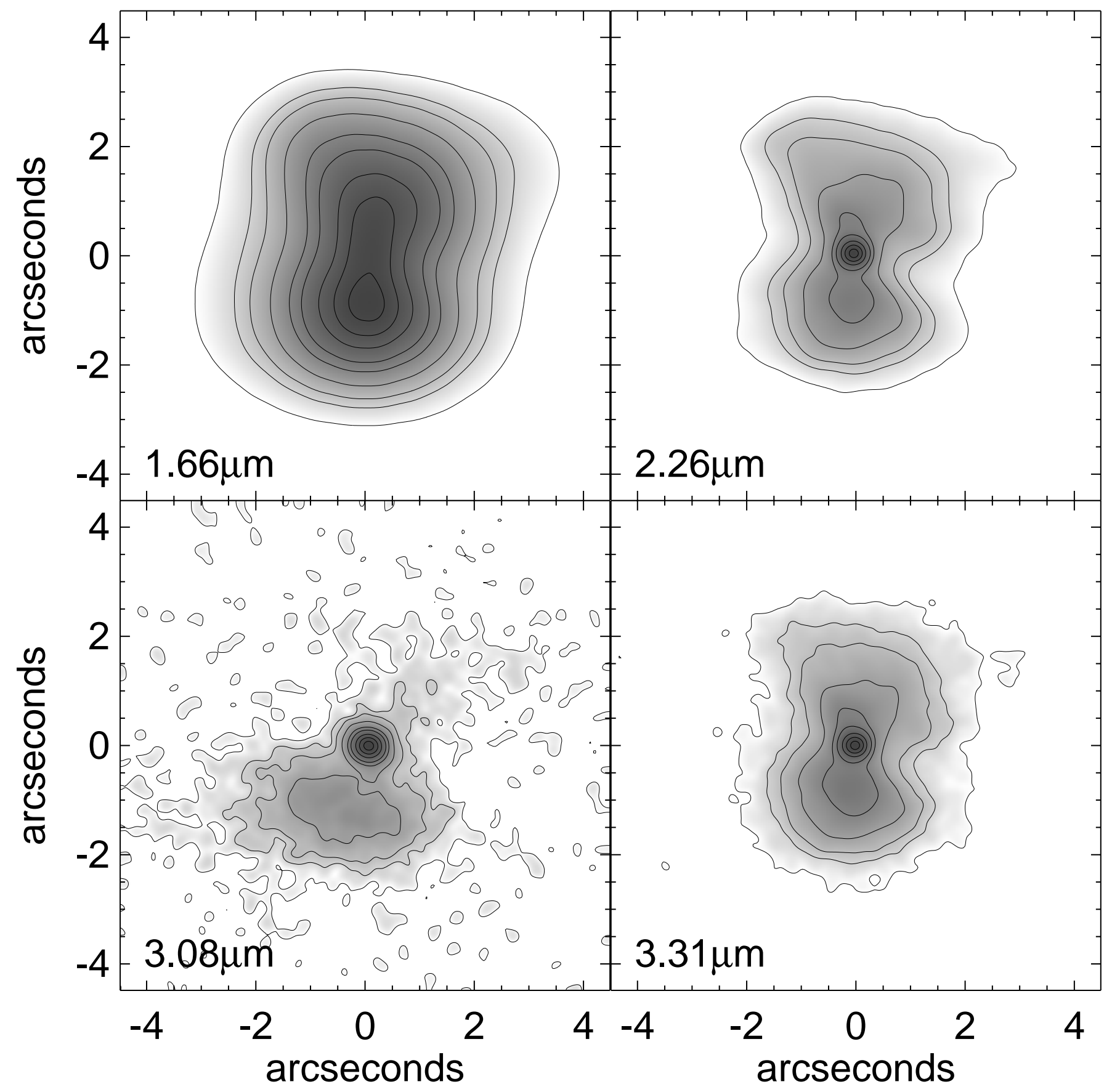

Contours (\% of Peak): $1 \quad 3 \quad 5 \quad 1020 \quad 3050 \quad 7090$

Fig. 1. Images of Frosty Leo at four wavelengths in the near-IR. The upper left panel contains an $H$-band image $\left(\lambda_{0}=1.66 \mu\right.$ m; $\Delta \lambda=0.33 \mu \mathrm{m}$ ) taken 9 June 1998. (Note: a camera error resulted in the UV blocking filter, used with the $H$ filter, to be absent for this observation.) The three remaining maps were all taken 7 June 1998 with the filters $\lambda_{0}=2.26 \mu \mathrm{m} ; \Delta \lambda=0.05 \mu \mathrm{m}$ (upper right), $\lambda_{0}=3.08 \mu \mathrm{m} ; \Delta \lambda=0.10 \mu \mathrm{m}$ (lower left), and $\lambda_{0}=3.31 \mu \mathrm{m} ; \Delta \lambda=0.06 \mu \mathrm{m}$ (lower right). North is up and East to the Left.

the bipolar lobes characteristic of Frosty Leo, does not show the bright central component visible at all other wavelengths. It seems likely that the increased optical depth through the dust shell at the shorter wavelength, together perhaps with enhanced scattering brightness of the lobes, have resulted in the central star at the heart of the nebula being completely obscured. The lack of a central point-component results in apparently larger lobes; an artifact due to the fact that contours are plotted in terms of the fraction of the peak flux. 


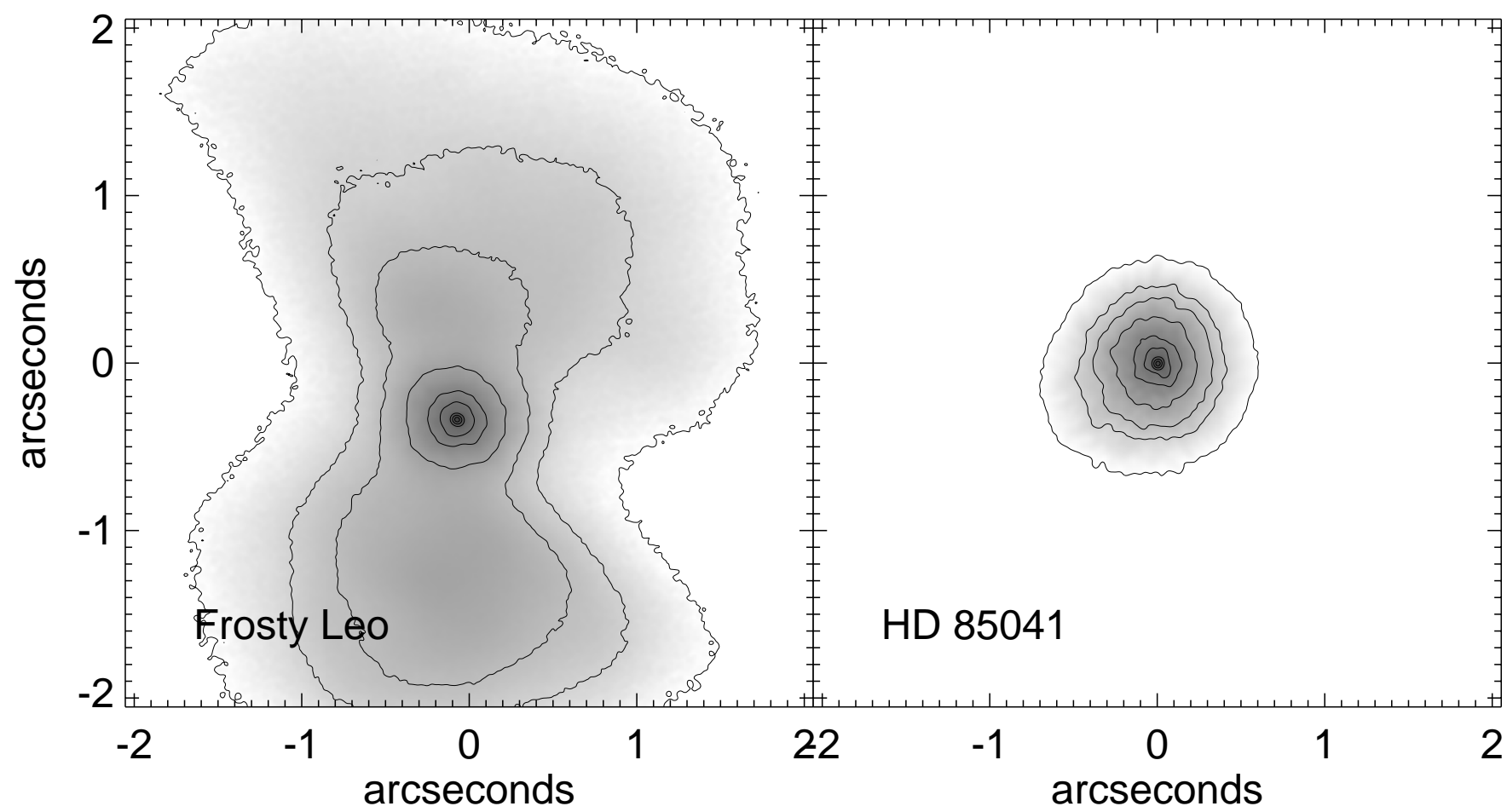

Contours (\% of Peak): 135102030507090

Fig. 2. $K$-band images $\left(\lambda_{0}=2.21 \mu \mathrm{m} ; \Delta \lambda=0.43 \mu \mathrm{m}\right)$ of Frosty Leo (Left) and the nearby point-source HD 85041 (Right) reconstructed with the shift-and-add algorithm from data taken on 7 June 1998. North is up and East to the Left.

The central star is apparent in the three remaining images in Fig. 1 straddling the ice band, with the $2.26 \mu \mathrm{m}$ towards short wavelength, the $3.08 \mu \mathrm{m}$ at the center, and $3.31 \mu \mathrm{m}$ in the continuum longwards of the absorption peak. Although the 2.26 and $3.31 \mu \mathrm{m}$ maps show remarkable similarity to each other, a dramatic extinction of the Northern lobe is clearly visible in the $3.08 \mu \mathrm{m}$ map centered on the ice band. The Southern lobe is slightly brighter in all maps, but the ratio of peak brightness of the South-North changes from a value close to 1 , to greater than 10 as the observing wavelength passes through $3.08 \mu \mathrm{m}$. Other more minor changes of morphology are apparent from Fig. 1, the visible Southern lobe being somewhat elongated E-W and more detached from the central source, as compared with either nearby continuum map. This bipolar/monopolar behavior is further discussed in the context of radiative transfer modeling in the following sections.

Capitalizing on the ability to resolve structure at the diffraction limit of the $10 \mathrm{~m}$ Keck aperture, a second set of observations was targeted at imaging the innermost regions of the nebula at high angular resolution. For this work, the image scale on the array was magnified by a factor of 7.29 resulting in a field size of $5{ }^{\prime \prime} 27$ - sufficient to sample the high-resolution image structure. Data sets consisted of 100 rapid-exposure ( $\sim 140 \mathrm{~ms})$ frames taken on Frosty Leo, followed by 100 frames on a nearby point-source star for calibration purposes.
This follows fairly standard speckle interferometry procedures, and there were a number of analysis options open in reducing such data into science quality images. An array of Fourier techniques for computing the bispectrum can be used in concert with statistically based deconvolution algorithms (such as the maximum entropy method) to reconstruct images, as has been successfully demonstrated for near-IR Keck data recently (Monnier et al. 1999; Tuthill et al. 1999; Tuthill et al. 2000). However for the case of Frosty Leo, which is highly resolved, and consequently has very low visibilities on all but the shortest baselines, the much simpler shift-and-add technique (Cady \& Bates 1980) has been found to deliver good imaging performance while avoiding the computational complexity of the Fourier methods.

We present in Fig. 2 the results of our shift-and-add analysis of Frosty Leo and of the nearby point-source star HD 85041. Examination of the HD 85041 picture shows that the shift-and-add technique recovers images with some $8 \%$ of the flux in a diffraction-limited core, with the remaining flux in a seeing-disk size halo. Although this is a modest recovery of the diffraction limited performance (as compared, for example, to adaptive optics) it is easily sufficient to study the high resolution structure of the inner nebula at limited dynamic range. It can be seen, from examination of the Frosty Leo image of Fig. 2, that at the epoch of our observations no secondary component or companion is visible in the inner region, which appears to 
be a single point source at this wavelength. Specifically, we find no evidence supporting the finding of a binary of nearly equally bright components with a separation of $0.19^{\prime \prime}$ as was observed in 1994 by Roddier et al. (1995). A faint companion beyond the limited dynamic range of our experiment might exist, however our observations place a limit of $\Delta M=2.5$ for any such component at the separation found by Roddier et al. (1995).

\section{Numerical simulations}

With the use of numerical simulation of radiative transfer through the dusty envelope, our aim is to define a set of model parameters that reproduce the general behaviour of the images presented in the above section together with the broad band spectrum. Although Frosty Leo is known to display a wealth of detail (e.g. bipolar jets, binarity), our focus here is on determining the physical conditions required to produce the general form of the $1.6,2.2,3.1$ and $3.3 \mu \mathrm{m}$ images.

\subsection{Radiative transfer code}

Radiative transfer through the envelope was computed by Monte Carlo numerical simulation, establishing a selfconsistent thermal profile for the dust in radiative equilibrium with the central star. This method has been successfully applied to numerous spherically symmetric problems (see for example Lefèvre et al. 1982) and more recently is being used to investigate axisymmetric dust nebulae (Lopez \& Perrin 2000).

Solving for the general case where grain absorption and (multiple) scattering may be strong functions of wavelength leads to situations where the optical appearance of the whole dust envelope can inherit these dependencies. Grains are assumed to be spherical (radius $a$ ) and isotropic, with the composition for Frosty Leo consisting of a silicate core (astronomical silicates, see Draine 1985) surrounded by a crystalline ice mantle (Bertie et al. 1969). The core of silicate represents half the volume of the grain, which corresponds to a mass fraction of water ice of about $40 \%$. Model grains range in size between $0.1 \mu \mathrm{m}<a<5.0 \mu \mathrm{m}$ with a size distribution of $a^{-3.5}$. These grains have been found to provide a good fit to the broadband spectrum and to its absorption band at $3.1 \mu \mathrm{m}$. For practical reasons the optical grain properties were averaged over the power law size distribution (a unique grain with averaged optical properties is considered in the code). While the equilibrium temperatures of grains of different sizes located at the same distance from the star are expected to differ, the use of a grain with mean optical properties leads to a mean value for the temperature.

The extinction $\left(C_{\text {ext }}\right)$, scattering $\left(C_{\text {sca }}\right)$ and absorption $\left(C_{\text {abs }}\right)$ cross sections, and also the angular dependence of the scattered radiation field with respect to the incident

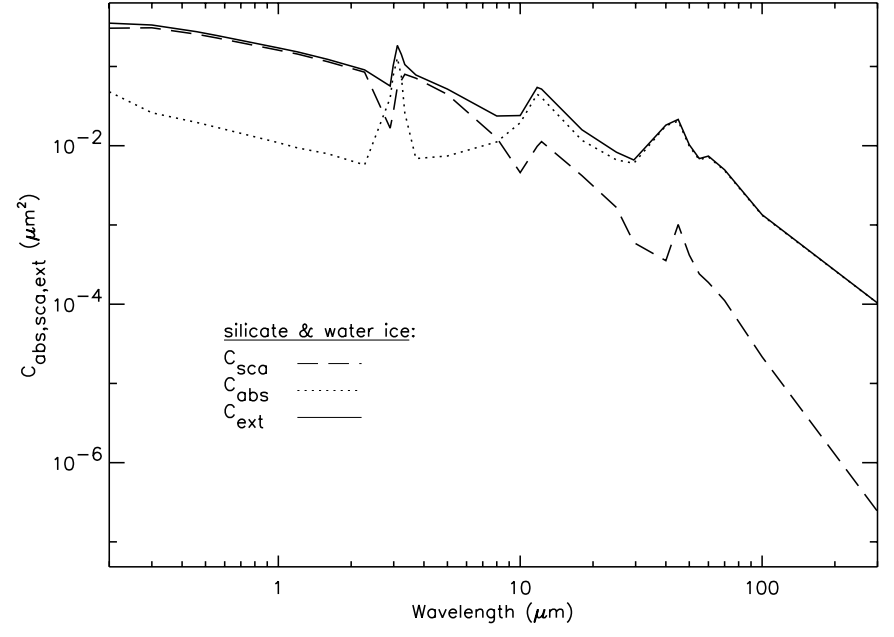

Fig. 3. Mean optical properties for the grain size distribution used in our models.

radiation field were calculated for spherical coated spheres (Aden \& Kerker 1951). Values of these mean cross-sections for the grain size distribution as a function of wavelength are given in Fig. 3 .

The spatial distribution of dust grains is an axisymmetric function $D(r, \delta)$, with $\delta$ being the "latitude" or angle from the plane of the disk, and $r$ the radial distance from the center of the star (in units of the photospheric radius). The function $D(r, \delta)$ is bounded by inner and outer limits denoted $R_{\text {in }}$ and $R_{\text {out }}$ respectively, outside which the density goes to zero. The central star is assumed to radiate as a blackbody with effective temperature $T_{\text {eff }}=3750 \mathrm{~K}$ (Robinson et al. 1992). The angular size of the central star, $\alpha_{*}$ is determined empirically by adjusting the model spectrum level to that observed. The radius of the star, $R_{*}$, and its distance, $D$, come into play in the simulations only via their ratio $\alpha_{*}$. Therefore no unit for $R_{*}$ needs to be specified and for simplicity we have set $R_{*}=1$ hereafter.

The model dust shell is gridded in about 400 tori, each of which has constant grain temperature from axisymmetric arguments. The Monte Carlo method proceeds by examination of large sets of random photon trajectories computed over thirty wavelength increments to represent the emergent spectrum of the stellar and dust radiation. Radiative exchange between the star and dust tori and between different tori maintain radiative equilibrium. Assuming negligible sublimation (or other chemical loss of heat), the temperature of each torus can be determined by balancing the absorbed and radiated energy. The global brightness distribution of the object (as a function of wavelength and inclination angle of the nebula) as well as its broad band spectrum (also a function of inclination angle) are obtained for a given set of model parameters. These model parameters are then varied empirically until the model maps match the form of the data images, and the spectrum is satisfactorily reproduced. 


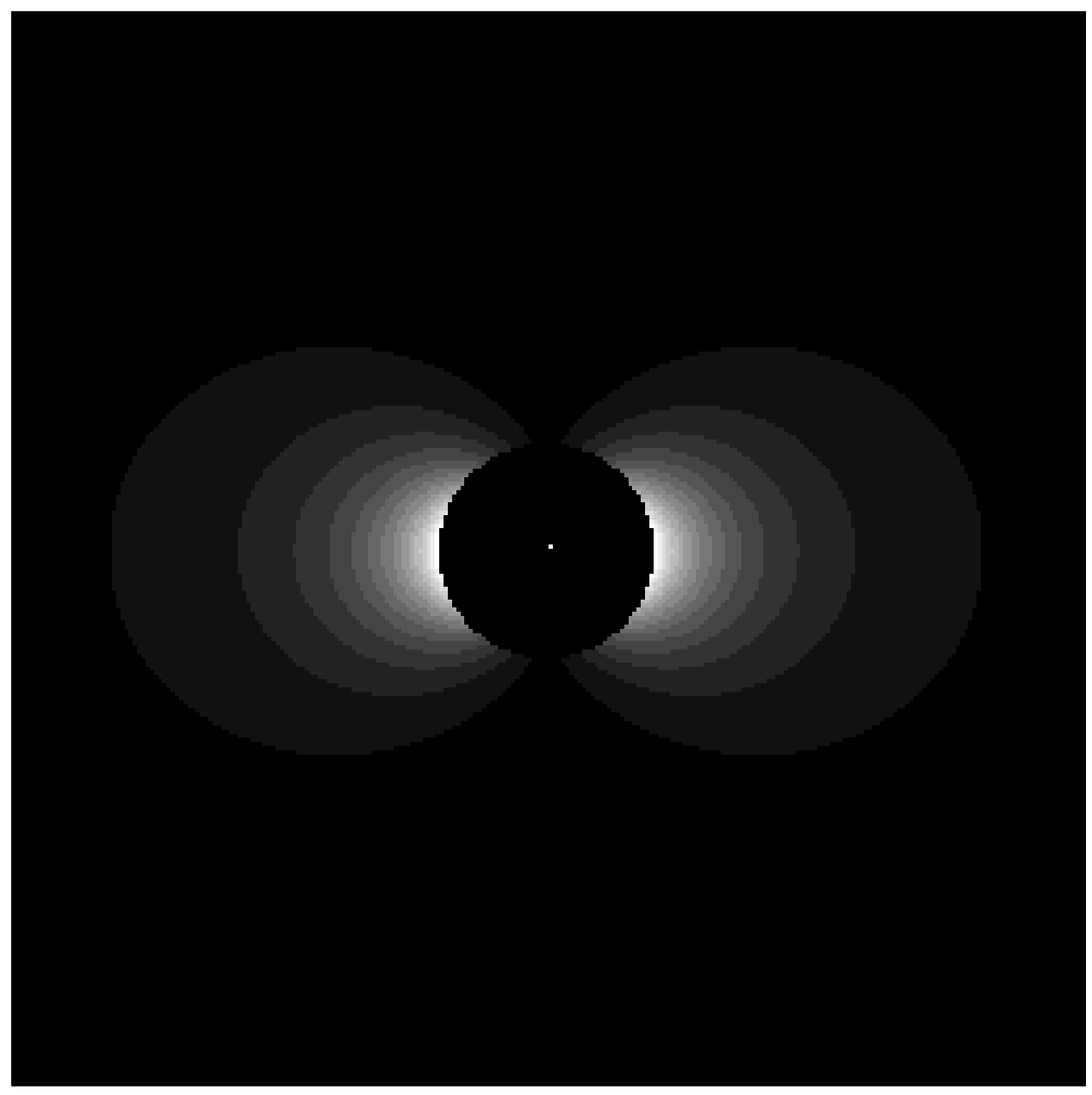

Fig. 4. The dust distribution $D(r, \delta)$ for the plane symmetric geometry. $D(r, \delta)$ is equal to $f(\delta) \times r^{-2}$ (at $r=2 \times R_{\text {in }}$ the dust density is 4 times smaller than in $\left.R_{\text {in }}\right)$. $f(\delta)$ consists of a Gaussian-profile density enhancement to the equatorial regions. The sigma of this Gaussian is $40^{\circ}$. The position of the star is marked with a dot.

\subsection{An axisymmetric model with reflection symmetry in the plane of the disk}

The general characteristics of several bipolar nebulae have been explained with models based on axisymmetric dust density laws with mirror symmetry with respect to the plane of the disk. Judging from the symmetrical lobes presented in the quasi-continuum maps, this appears to be an encouraging place to start. One of the most intriguing features, and most difficult to reproduce with the modeling, is the almost total disappearance of the Northern lobe at $3.1 \mu \mathrm{m}$. The most straightforward explanation for this is that the observer's line-of-sight to the Northern lobe must traverse an equatorial disk rich in water ice. In this hypothesis, the Southern side of the disk is tilted towards the observer, obscuring the Northern lobe.

The first model we present consisted of a thick disk, tilted to give a clearer line of sight to the Southern lobe. The disk was modeled as a Gaussian-profile density enhancement in the equatorial regions. The optically thin polar regions allow efficient scattering of stellar radiation
Table 1. Model parameters used in the simulations.

\begin{tabular}{cccccc}
\hline$T_{\text {eff }}(\mathrm{K})$ & $\alpha_{*}(\mathrm{mas})$ & $R_{\text {in }}\left(R_{*}\right)$ & $R_{\text {out }}\left(R_{*}\right)$ & $a_{\text {grains }}(\mu \mathrm{m})$ & $\tau_{\text {ext }}^{*}$ \\
\hline 3750 & 0.3 & $2.3 \times 10^{3}$ & $3 \times 10^{4}$ & $0.1<a<5.0$ & 11. \\
\hline
\end{tabular}

* Optical depth at $1 \mu \mathrm{m}$ and for $\delta=0^{\circ}$.

by dust particles in the lobes of the nebula. In the radial direction, the flow followed a simple $r^{-2}$ outflow law. In the plane of the disk the optical depth at $1 \mu \mathrm{m}$ was 11 , causing the narrow equatorial waist visible in the images. The density law $D(r, \delta)$ is shown in Fig. 4, while other basic model parameters can be found in Table 1 .

Model image profiles generated by radiative transfer through this geometry are given in Fig. 5, which shows a set of simulated images at $3.106 \mu \mathrm{m}$ for different inclination angles of the disk with respect to the line of sight. Many of the features of Frosty Leo are reproduced well by this simple model including the quasi-continuum images and broadband spectrum between 0.20 to about $100 \mu \mathrm{m}$ 


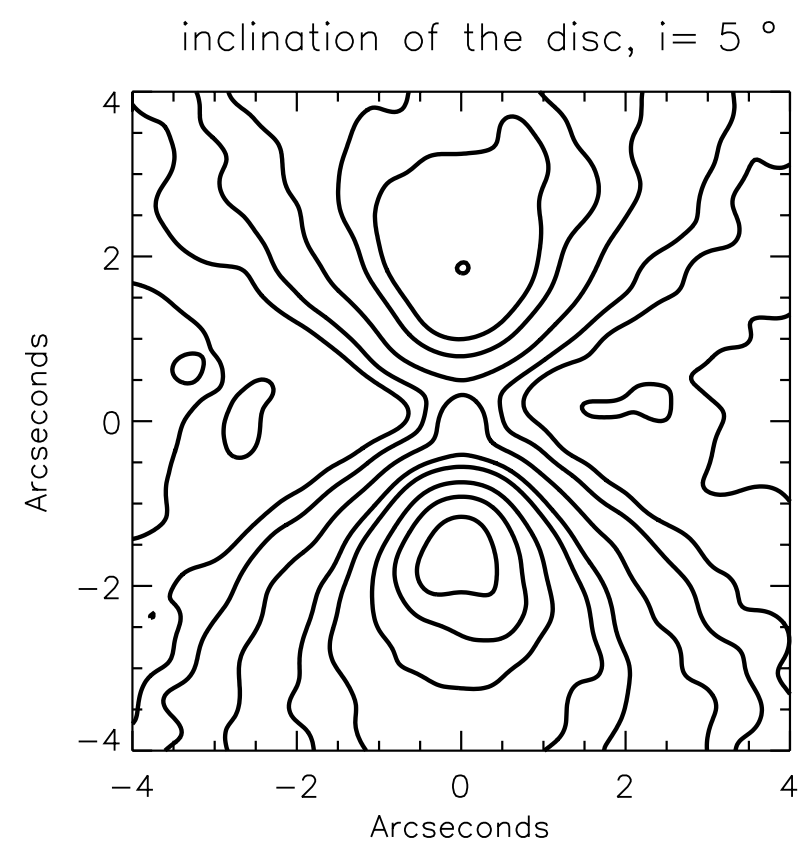

inclination of the disc, $i=16^{\circ}$

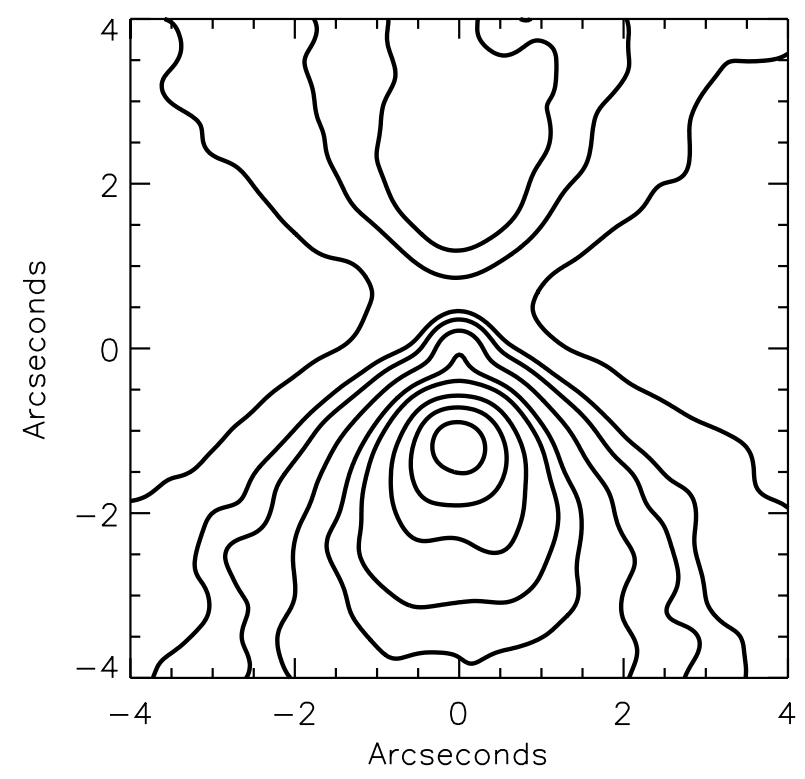

inclination of the disc, $i=10^{\circ}$

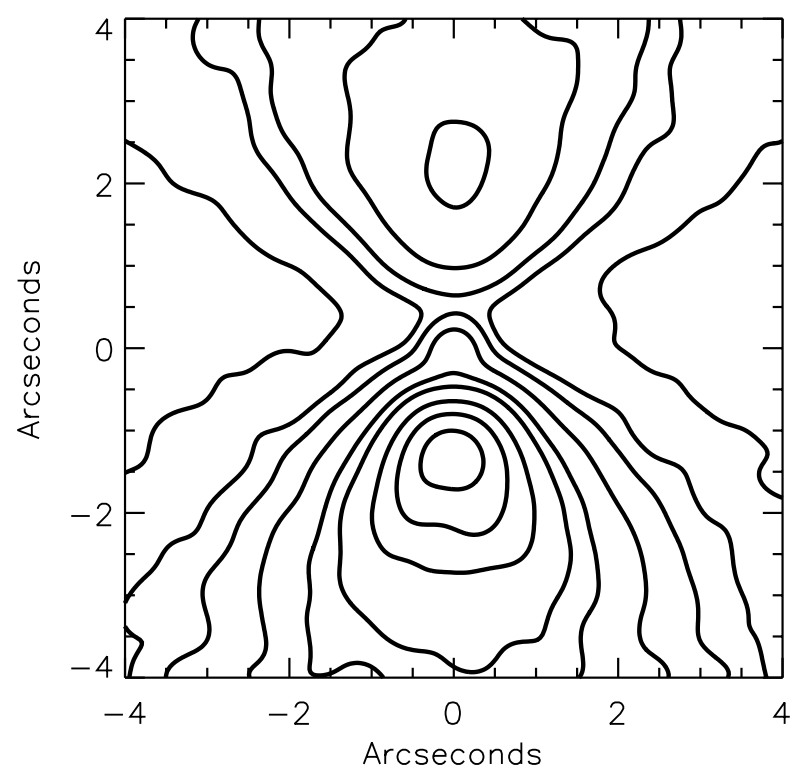

inclination of the disc, $i=22^{\circ}$

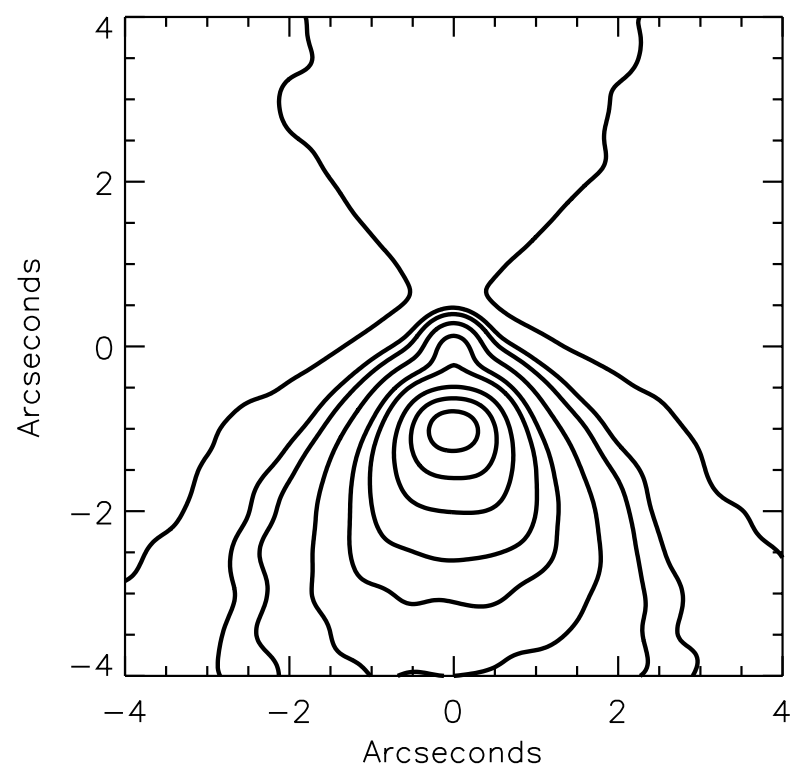

Fig. 5. Simulated images at $3.1 \mu \mathrm{m}$ of the symmetric disk model displayed for different inclinations of the disk. This simulated image is convolved by a Gaussian with a width at half maximum of $0.5^{\prime \prime}$. The contour levels are at 1, 3, 5, 10, 20, 30, 50, 70, $90 \%$ of the peak intensity.

(not displayed as it is not significantly different to the spectrum shown in Fig. 6 described in the next section).

Despite the exploration of a large parameter space encompassing many density laws and various forms of global dust shell geometry, no completely satisfactory symmetric model was found which could reproduce the switch from bipolar to highly monopolar through the water-ice band, while simultaneously preserving a good match to the spectrum and maps at other wavelengths. The density law used here (Fig. 4) has the functional form $D(r, \delta)=D\left(R_{\text {in }}, 0\right) \times f(\delta) \times r^{-2} \cdot f(\delta)$ consists of a Gaussian-profile density enhancement to the equatorial regions, the sigma of this Gaussian is $40^{\circ}$. We have played with the parameters of the density law by varying $D\left(R_{\text {in }}, 0\right)$ (in our simulation, $D\left(R_{\text {in }}, 0\right)$ is constrained through the optical depth of the disk which is the input parameter), $R_{\text {in }}, R_{\text {out }}, \delta$ and $i$, the inclination of the disk. Other functional relationship were used. A cut in the above density law for $|\delta|>45^{\circ}$ giving $f\left(|\delta|>45^{\circ}\right)=0$ was tested. Another relationship linearly decreasing from 

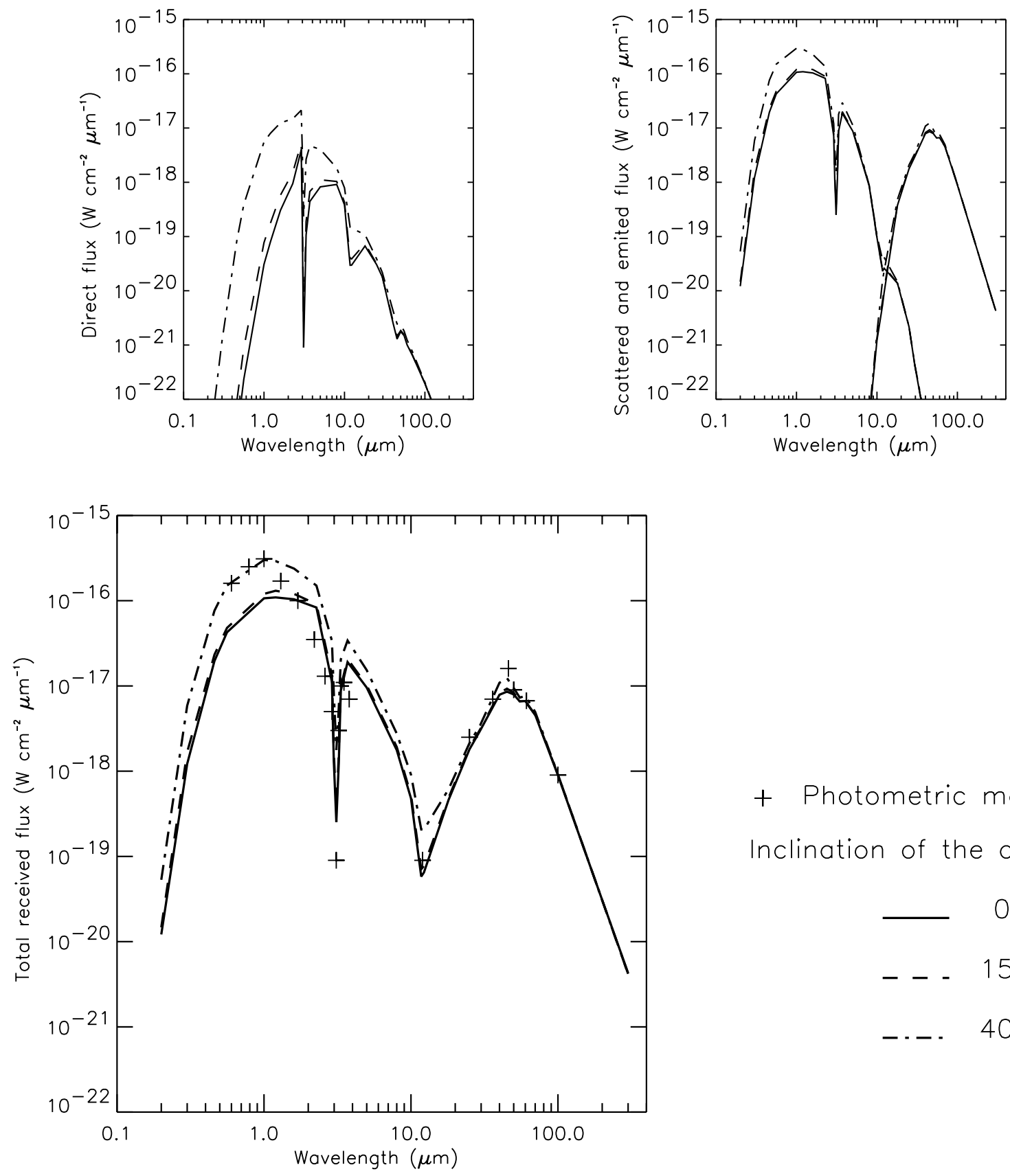

+ Photometric measurements

Inclination of the disc-model :

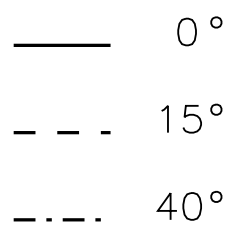

Fig. 6. Simulated spectrum of the proposed asymmetric model for different inclinations of the disk. The spectrophotometric data come from a compilation of Robinson et al. (1992).

$\delta=0^{\circ}$ to $\delta=90^{\circ}$ was also tested. By changing the density laws, no significant improvement has been obtained in the data fitting. Playing with the parameters of the laws was carefully done for the gaussian relationship $D(r, \delta)=D\left(R_{\text {in }}, 0\right) \times f(\delta) \times r^{-2}$, less carefully done for the other functional forms.

\subsection{A model with an asymmetric structure with respect to the plane of the disk}

In an attempt to overcome the problems with matching the single-lobed appearance at $3.1 \mu \mathrm{m}$, we investigated models in which the reflection symmetry through the plane of the disk was broken. The best results have been obtained with the dust envelope whose functional form $D(r, \delta)$ is displayed in Fig. 7. Similar in many respects to the earlier model, the reflection symmetry between the lobes has now been broken by the faster decrease of $D(r, \delta)$ in the South lobe of the nebula.

Our model was found to provide a reasonably good fit to the observations. Fits to the broadband spectrum (Fig. 6) were again good with thermal emission from dust dominating at long wavelengths $(\gtrsim 10 \mu \mathrm{m})$ while scattering, more important at shorter wavelengths, is responsible for the bright bipolar lobes. Simulated images at 1.600, 


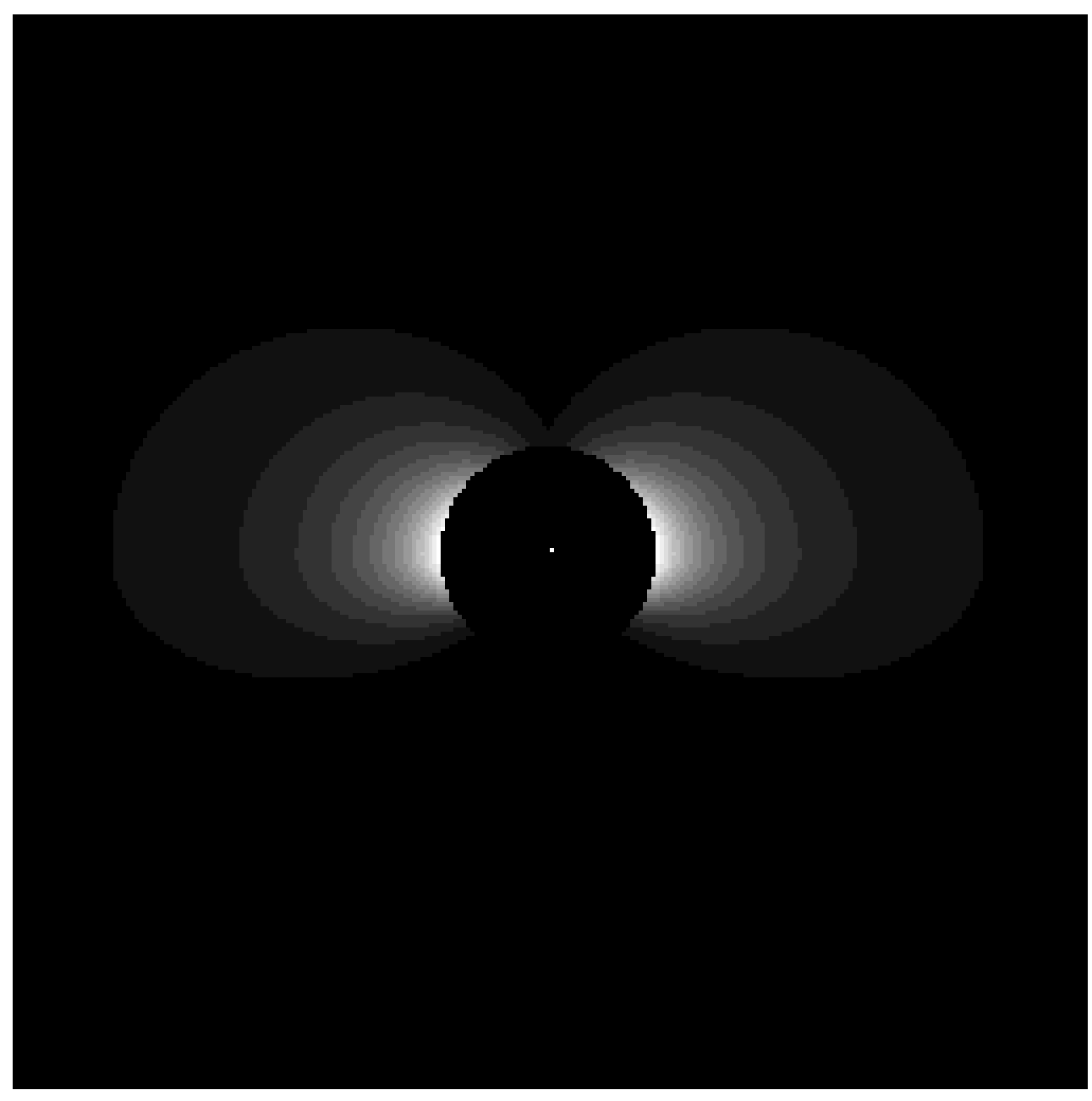

Fig. 7. The dust distribution $D(r, \delta)$ for the asymmetric geometry regarding to the plane of the disk. $D(r, \delta)$ is equal to $f(\delta) \times r^{-2}$. $f(\delta)$ consists of a Gaussian-profile density enhancement to the equatorial regions. The sigma of this Gaussian is $40^{\circ}$ for $\delta>0^{\circ}$, and sigma $=20^{\circ}$ for $\delta<0^{\circ}$. The position of the star is marked with a dot.

$2.273,3.106$ and $3.333 \mu \mathrm{m}$ are shown in Figs. 8-11 respectively. Each figure includes different inclinations of the plane of the disk $\left(5,10,16,22^{\circ}\right)$ with respect to the line of sight. Under the assumptions of our model, the morphology of Frosty Leo appears reasonably well reproduced for an inclination angle around $16^{\circ}$. The brightness ratio of the lobes in the $3.106 \mu \mathrm{m}$ simulated image, formerly entirely due to viewing angle, is now enhanced by the unbalanced density profile, resulting in a value of about 10 . This brightness ratio between the lobes is very close to that observed in the $3.08 \mu \mathrm{m}$ observations. Moreover, in this model the star appears more prominent than in the $3.106 \mu \mathrm{m}$ image corresponding to the plane symmetrical model.

\section{Discussion and conclusions}

A near-infrared imaging study of the spatial distribution of $\mathrm{H}_{2} \mathrm{O}$ ice in Frosty Leo performed with the Keck telescope has yielded a number of interesting results. Images have been made at a wavelength of $3.08 \mu \mathrm{m}$ within the prominent (attenuation of about $5 \mathrm{mag}$ ) water-ice absorption band centered around $3.1 \mu \mathrm{m}$, in addition to quasi-continuum maps at adjacent wavelengths of $1.6 \mu \mathrm{m}$, $2.2 \mu \mathrm{m}$, and $3.3 \mu \mathrm{m}$. Based on these data, and also upon previously-published broadband spectral data, the question of the physical structure of the nebula has been addressed with the use of numerical radiative transfer simulations. We have limited our investigation to a small number of the simplest possible geometries with an emphasis on reproducing the general behaviour of the observed images, and in particular, the monopolar image observed at $3.08 \mu \mathrm{m}$. A more detailed modelling study in progress (Niccolini et al. 2001) aims at understanding what the role played by each model parameter and the accuracy with which the parameters may be estimated.

One of the major difficulties encountered in modeling the circumstellar nebula was reproducing the one-sided lobe at $3.08 \mu \mathrm{m}$, which would seem to have its origin in a greater optical depth in the water-ice band to the Northern lobe from the observer's viewing angle. Although 
inclination of the disc, $i=5^{\circ}$

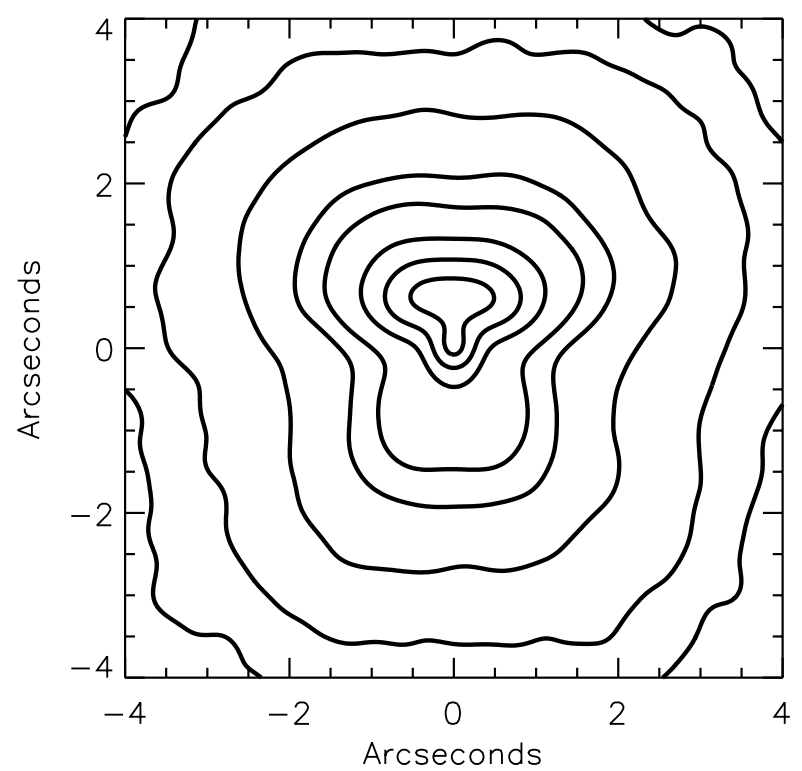

inclination of the disc, $i=16^{\circ}$

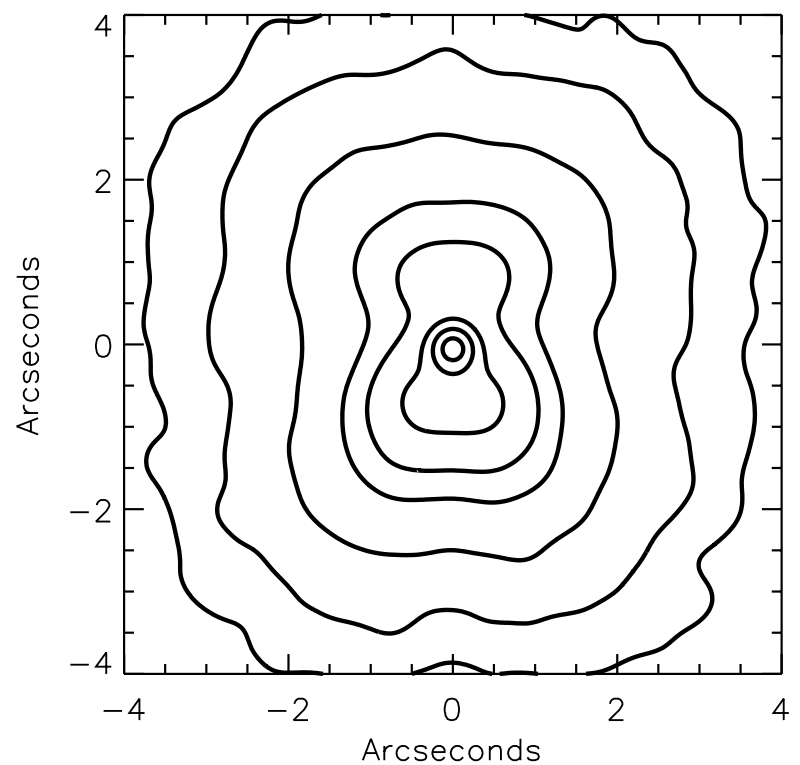

inclination of the disc, $i=10^{\circ}$

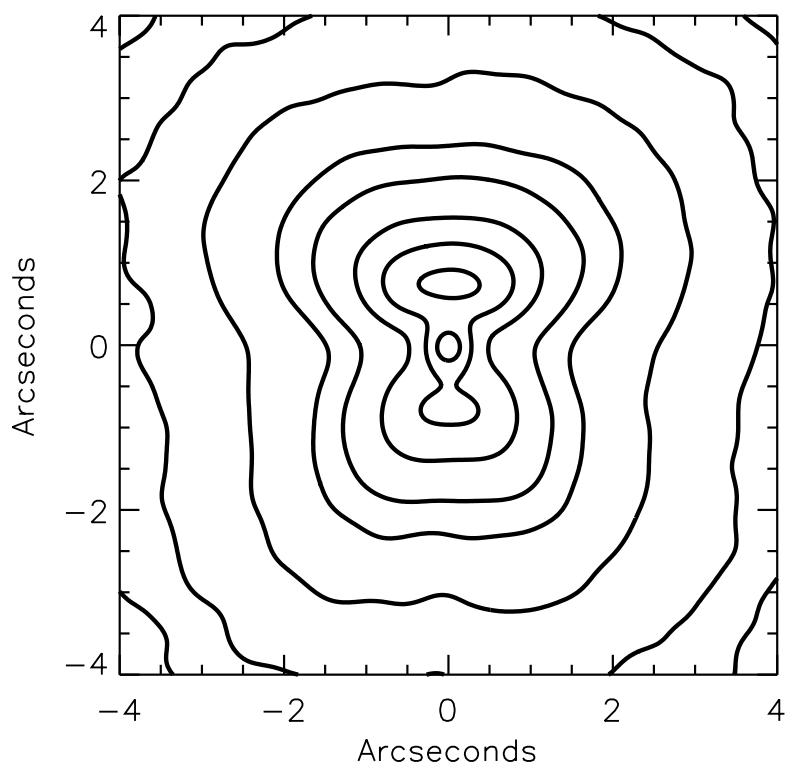

inclination of the disc, $i=22^{\circ}$

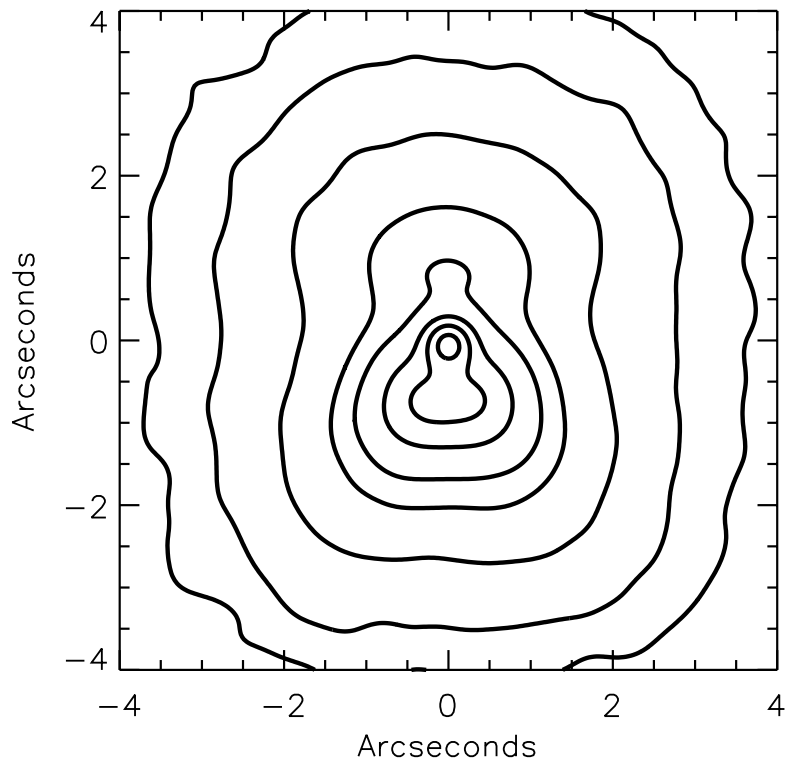

Fig. 8. Simulated images at $1.6 \mu \mathrm{m}$ displayed for different inclinations of the asymmetric disk model. This simulated image is convolved by a Gaussian with a width at half maximum of $0.5^{\prime \prime}$. The contour levels are at $1,3,5,10,20,30,50,70,90 \%$ of the peak intensity.

models with symmetrical lobes (with regard to the plane of the disk) could generate significant asymmetric extinction through choice of a viewing angle preferring one lobe over the other, no fully satisfactory solutions were found.

A small number of models with an asymmetric density structure with respect to the plane of the disk were also considered. Such models give a better match to the images, generating larger extinction ratios between the bright and faint lobe observed at $3.08 \mu \mathrm{m}$. Chemical difference between the lobes (which we are not able to simulate with the present numerical code), or even more subtle grainsize differences (which can have a large impact on the optical properties) may also be worthy of consideration as alternate candidates for producing the monopolar shape at $3.08 \mu \mathrm{m}$.

Of course, these somewhat ad-hoc solutions come at the expense of extra free model parameters. One of the alternative models, providing some interesting results and tested with simulations, is an asymmetric geometry with a disk which is twice as high on the Northern side compared to the Southern one. 

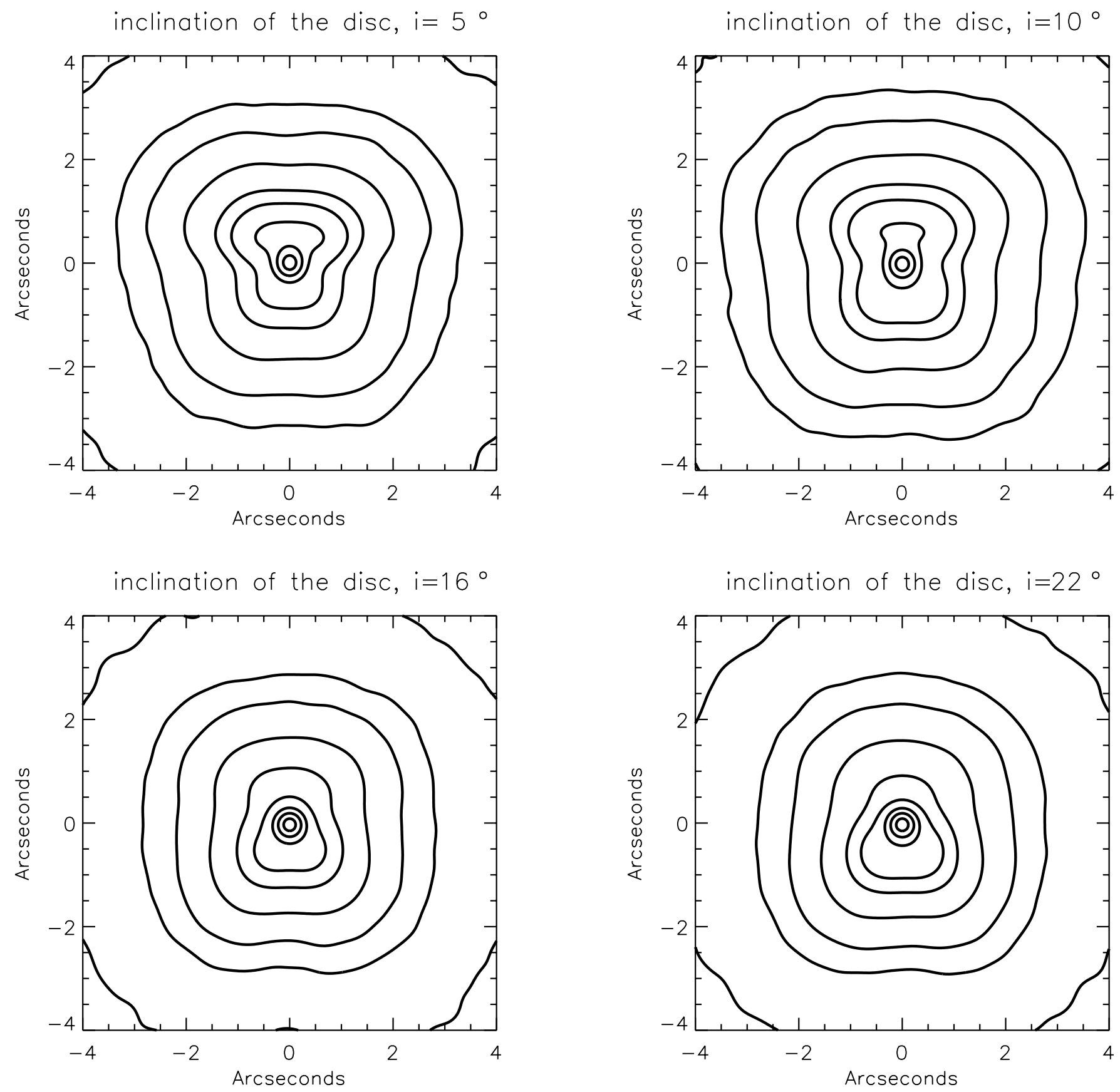

Fig. 9. Simulated images at $2.2 \mu \mathrm{m}$ displayed for different inclinations of the asymmetric disk model. This simulated image is convolved by a Gaussian with a width at half maximum of $0.5^{\prime \prime}$. The contour levels are at 1, 3, 5, 10, 20, 30, 50, 70, 90\% of the peak intensity.

Models presented based on such thick equatorial disks have been able to reproduce the general behavior of the images and spectral data. The Frosty Leo morphology appears well reproduced for an inclination angle around $16^{\circ}$. In our model the Southern side is tilted towards the observer, in contrast to the proposed orientation of Roddier et al. (1995).

From the models, we have been able to estimate some of the physical properties of Frosty Leo. Both the models presented here, and those of Robinson et al. (1992), have a radius of the inner cavity, outside of which ice appears, of order $0.8^{\prime \prime}$. Temperatures of the hottest dust grains were found to be around 80-90 K. This compares well with previous work based on a spherically symmetric model constrained by the broadband spectrum (Robinson et al. 1992). In this range of temperature, according to Kouchi et al. (1994) theoretical and experimental results indicate that $\mathrm{H}_{2} \mathrm{O}$ is expected to be crystalline at the time of condensation. 

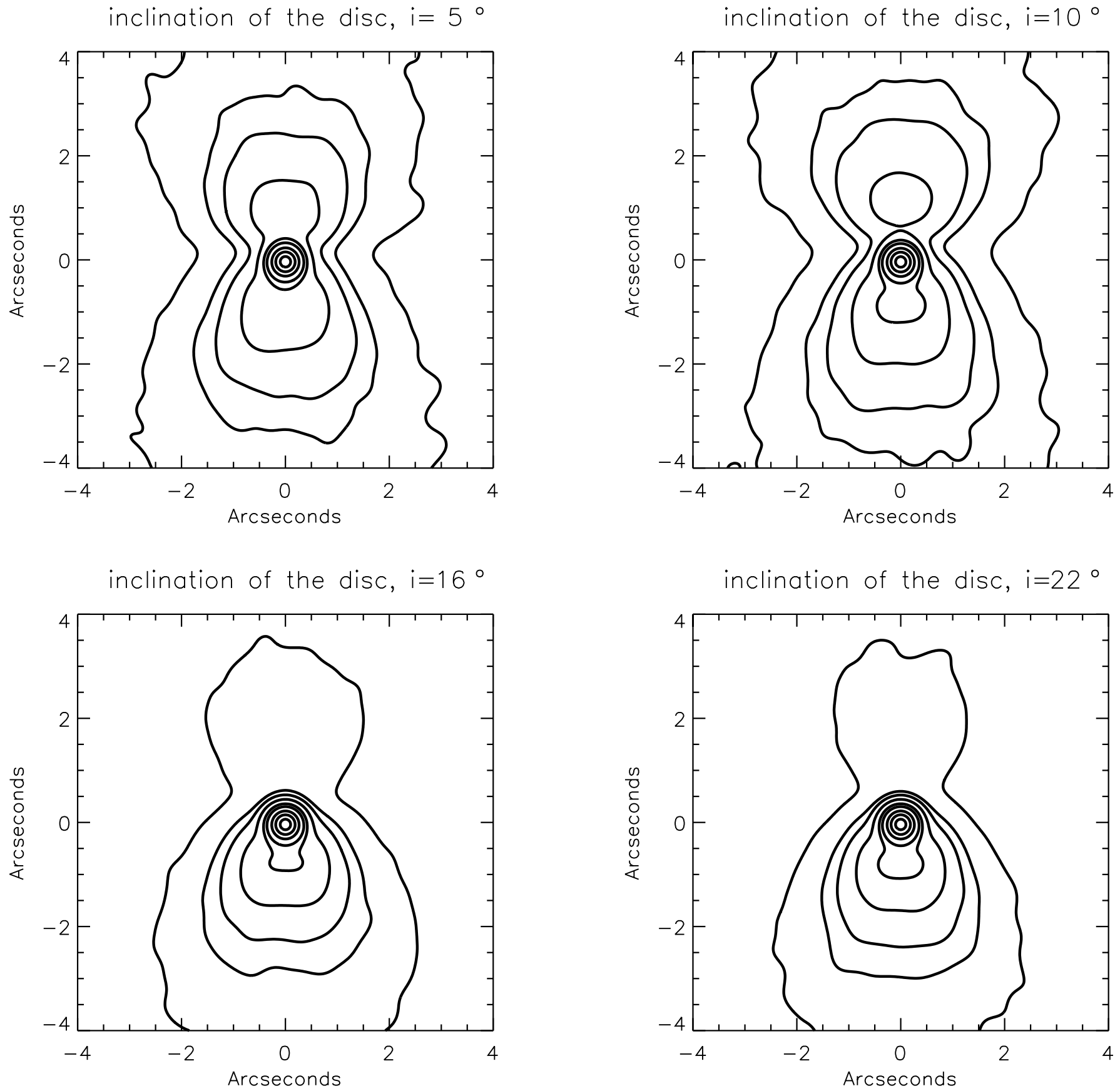

Fig. 10. Simulated images at $3.1 \mu \mathrm{m}$ displayed for different inclinations of the asymmetric disk model. This simulated image is convolved by a Gaussian with a width at half maximum of $0.5^{\prime \prime}$. The contour levels are at $1,3,5,10,20,30,50,70,90 \%$ of the peak intensity.

From Rouan et al. (1988) an upper limit on the dust mass in the $2^{\prime \prime}$ central region is $1.0 \times 10^{-4}[D / \mathrm{kpc}]^{2} M_{\odot}$. Our present estimate is that the total envelope mass in the case of the asymmetric model (of Sect. 3.3) is around $7.9 \times 10^{-4}[D / \mathrm{kpc}]^{2} M_{\odot}$ (assuming a density of the silicate material of $2 \times 10^{3} \mathrm{~kg} \mathrm{~m}^{-3}$ and a density for the water ice of $1 \times 10^{3} \mathrm{~kg} \mathrm{~m}^{-3}$ ).

We have not detected the almost equal-magnitude central binary star reported in Roddier et al. (1995) and have placed a (somewhat conservative) upper limit of $\Delta M=2.5$ for the relative magnitude of any extra component at the reported angular separation of $0.19^{\prime \prime}$.

Acknowledgements. Data presented herein were obtained at the W. M. Keck Observatory, which is operated as a scientific partnership among the California Institute of Technology, the University of California and the NASA. The Observatory was made possible by the generous financial support of the 
inclination of the disc, $i=5^{\circ}$

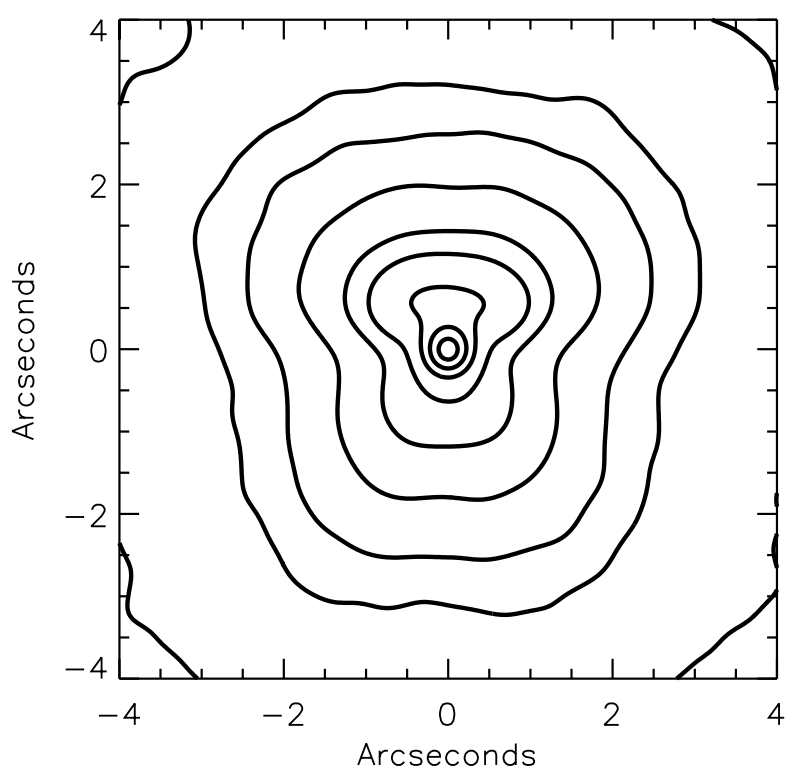

inclination of the disc, $i=16^{\circ}$

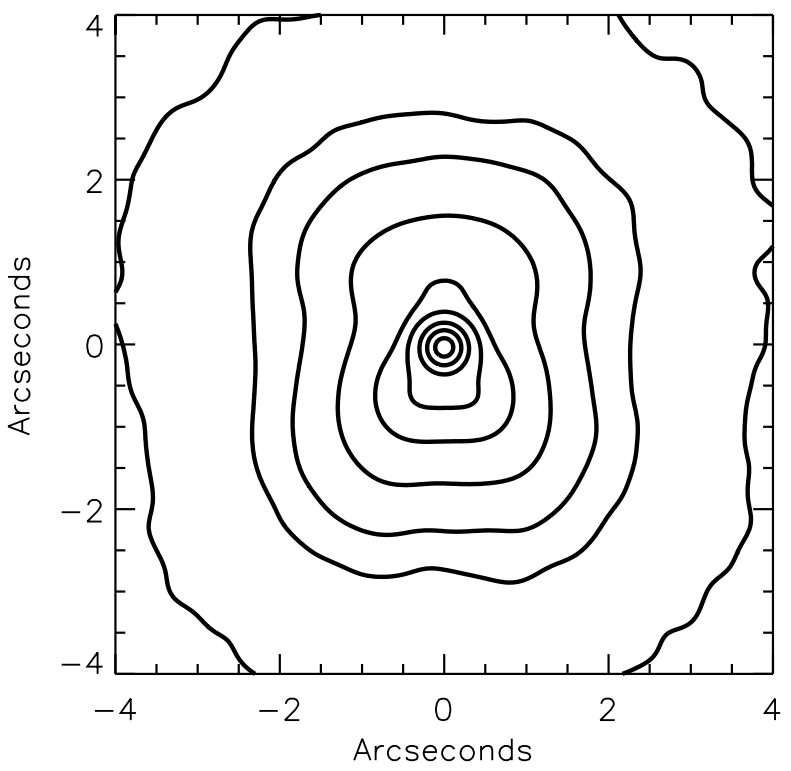

inclination of the disc, $i=10^{\circ}$

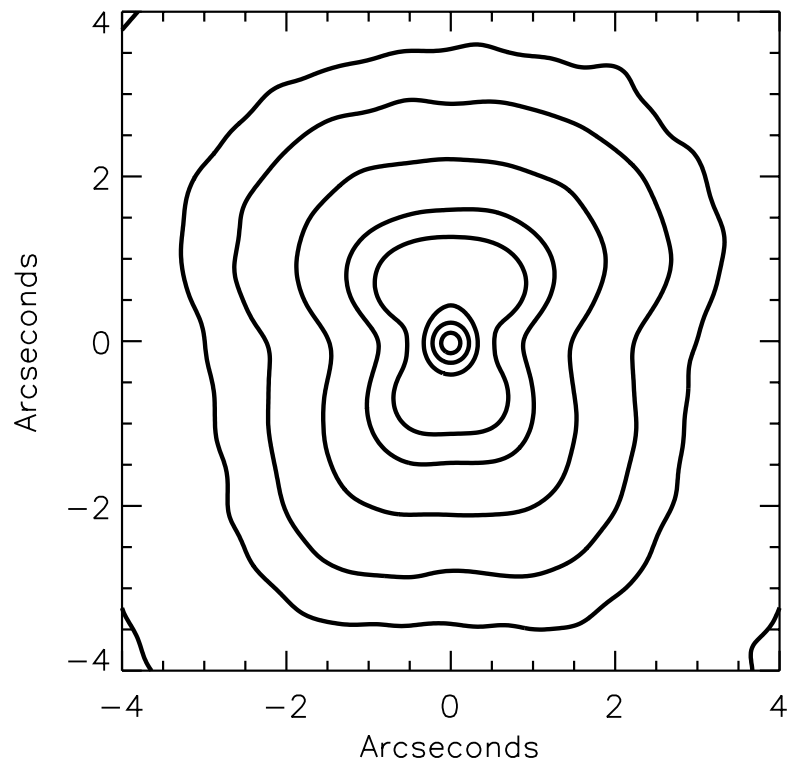

inclination of the disc, $i=22^{\circ}$

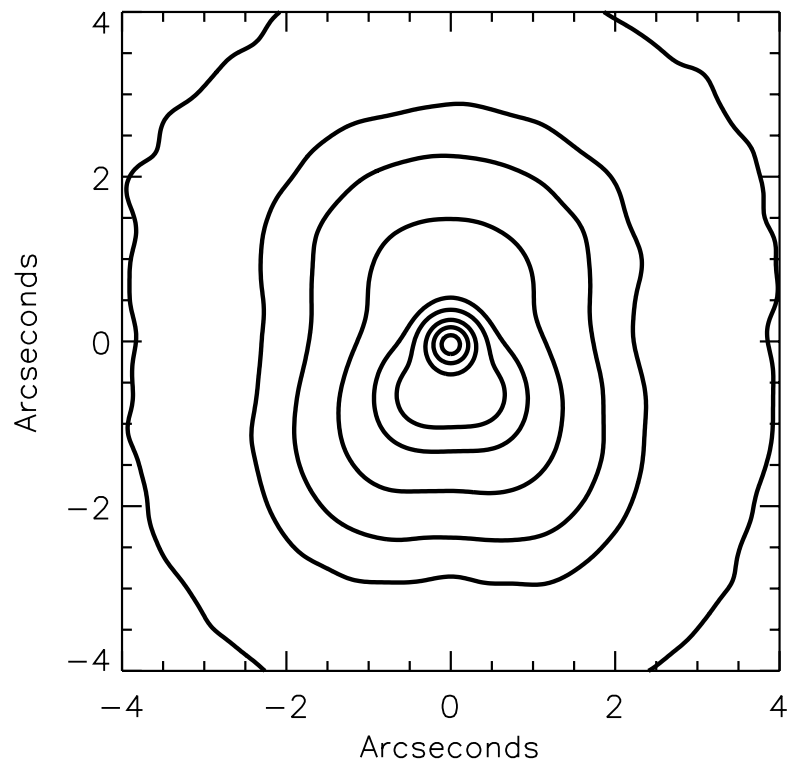

Fig. 11. Simulated images at $3.3 \mu \mathrm{m}$ displayed for different inclinations of the asymmetric disk model. This simulated image is convolved by a Gaussian with a width at half maximum of $0.5^{\prime \prime}$. The contour levels are at 1, 3, 5, 10, 20, 30, 50, 70, 90\% of the peak intensity.

W. M. Keck Foundation. We thank the anonymous referee for his contribution in improving this paper.

\section{References}

Aden, A. L., \& Kerker, M. 1951, J. Appl. Phys., 22, 1242 Alves, D. R., \& Hoard, D. W. 1996, AJ, 112, 230

Bertie, J. E., Labbé, H. J., \& Whalley, E. 1969, J. Chem. Phys., 50,4501

Blöker, T. 1995, A\&A, 299, 755

Cady, F. M., \& Bates, R. H. T. 1980, Opt. Lett., 5, 438
Draine, B. T. 1987, ApJS, 57, 587

Eiroa, C., Hefele, H., \& Qian, Z.-Y. 1983, A\&AS, 54, 309

Forveille, T., Morris, M., Omont, A., \& Likkel, L. 1987, A\&A, 176, L13

Iben, I. 1985, in Mass Loss from Red Giants, ed. M. Morris, \& B. Zuckerman (Dordrecht: Reidel), 1

Iben, I. 1991, ApJS, 76, 55

Kouchi, A., Yamamoto, T., Kozasa, T., et al. 1994, A\&A, 290, 1009

Rouan, D., Lacombe, F., Omont, A., \& Forveille, T. 1988, A\&A, 189, L3 
Lamy, P., \& Perrin, J. M. 1997, A\&A, 327, 1147

Lafon, J. P. J., \& Berruyer, N. 1991, A\&AR, 2, 249

Lefèvre, J., Bergeat, J., \& Daniel, J. Y. 1982, A\&A, 114, 341

Lopez, B., \& Perrin, J. M. 2000, A\&A, 354, 657

Likkel, L., Morris, M., Omont, A., \& Forveille, T. 1987, A\&A, 173, L11

Mastrodemos, N., \& Morris, M. 1998, ApJ, 497, 303

Mastrodemos, N., \& Morris, M. 1999, ApJ, 523, 357

Matthews, K., \& Soifer, B. T. 1994, Infrared Astronomy with Arrays: the Next Generation, ed. I. McLean (Dordrecht: Kluwer Academic Publishers), 239

Mauron, N., LeBorgne, J. F., \& Picquette, M. 1989, A\&A, 218, 213

Monnier, J. D., Tuthill, P. G., \& Danchi, W. C. 1999, ApJ, 525, 97

Morris, M. 1981, ApJ, 249, 572

Morris, M. 1987, PASP, 99, 1115

Morris, M., \& Reipurth, B. 1990, PASP, 102, 446

Niccolini, et al. 2001, A\&A, in preparation
Omont, A., Forveille, T., Moseley, S. H., et al. 1990, ApJ, 355, 270

Robinson, G., Smith, R. G., \& Hyland, A. R. 1992, MNRAS, 256, 437

Roche, P. F., \& Aitken, D. K. 1984, MNRAS, 209, 33

Roddier, F., Roddier, C., Graves, J. E., \& Northcott, M. J. 1995, ApJ, 443, 249

Rouan, D., Omont, A., Lacombe, F., \& Forveille, T. 1988, A\&A, 189, L3

Scarrot, S. M., \& Scarrott, R. M. J. 1994, MNRAS, 268, 615

Soifer, B. T., Willner, S. P., Rudy, R. J., \& Capps, R. W. 1981, ApJ, 250, 631

Tuthill, P. G., Haniff, C. A., \& Baldwin, J. E. 1999, MNRAS, 306,353

Tuthill, P. G., Monnier, J. D., Danchi, W. C., et al. 2000, PASP, 112,555

Van Winkels, H., Waelkens, C., \& Waters, L. B. F. M. 1995, A\&A, 293, L25

Yusef-Zadeh, F., Morris, M., \& White, R. 1984, ApJ, 278, 186 